\title{
Spectral multipliers \\ on metabelian groups
}

Waldemar Hebisch

\section{Introduction.}

Let $G$ be a Lie group, $X_{j}$ right invariant vector fields on $G$, which generate (as a Lie algebra) the Lie algebra of $G$,

$$
L=-\sum X_{j}^{2}
$$

Then $L$ is called sublaplacian, and it well-known that $L$ is positive definite and essentially selfadjoint on $C_{c}^{\infty}(G) \subset L^{2}(G)$, where $L^{2}(G)$ is taken with respect to a left-invariant Haar measure $d g$. By the spectral theorem, for any bounded Borel measurable function $F:[0, \infty) \longmapsto \mathbb{C}$ the operator $F(L) f=\int_{0}^{\infty} F(\lambda) d E(\lambda) f$ is bounded on $L^{2}(G)$. We are interested in the behavior of $F(L)$ on $L^{p}$.

This question has a long history. Classical results for polynomial growth case are [15], [16], [5], [18], [1], [7], [22] for exponential growth [8], [23], [2], [3]. Newer results show that connection with growth is more complicated [11], [19], [12], [10], [9], [4], [6], [13], [20], [14], [21], [17].

In this paper we consider $L^{1}(G)$ boundedness of $F(L)$ for (some) metabelian $G$ and a distinguished $L$ on $G$. Of the main interest is that the group is of exponential growth, and possibly higher rank. Previously positive results about higher rank groups where only about Iwasawa type groups. Also, our groups may be unimodular, so it is the second positive result (after [13]) about unimodular groups, and the first giving a family of examples. 


\section{Results.}

Let $G=\mathbb{R}^{n} \ltimes \mathbb{R}^{m}$, adjoint action is semisimple, $L=L_{0}+L_{1}, L_{0}$ lives on $\mathbb{R}^{n}, L_{1}$ lives on $\mathbb{R}^{m}$ and is a sum of (squares of) eigenvectors for adjoint action. More precisely, assume that $\lambda_{j}, j=1, \ldots, m$ are linear forms on $\mathbb{R}^{n}, e_{j}, j=1, \ldots, m$ is the canonical basis of $\mathbb{R}^{m}$, linear operator $A(x): \mathbb{R}^{m} \longmapsto \mathbb{R}^{m}$ is given by the formula $A(x) e_{j}=\lambda_{j}(x) e_{j}$ and

$$
\left(x_{1}, y_{1}\right)\left(x_{2}, y_{2}\right)=\left(x_{1}+x_{2}, \exp \left(A\left(-x_{2}\right)\right) y_{1}+y_{2}\right) .
$$

The right-invariant vector fields are:

$$
X_{j}=\partial_{x_{j}}
$$

and

$$
Y_{j}=\exp \left(-\lambda_{j}(x)\right) \partial_{y_{j}}
$$

We assume that

$$
L=-\sum X_{j}^{2}-\sum Y_{j}^{2}=L_{0}+L_{1}
$$

We can transform general $L_{0}$ to our form, but for $L_{1}$ the assumption is somewhat restrictive.

In this paper we identify convolution operators with functions:

$$
\exp (-t L) f=\exp (-t L) * f
$$

Theorem 1.1. If $G$ and $L$ are as above, then there exists $C$ such that

$$
\|\exp (-(1+i s) L)\|_{L^{1}} \leq C\left(1+|s|^{3 m+n}\right) .
$$

Thorem 1.2. For every compactly supported $F \in C^{3 m+n+1}$ the operator $F(L)$ is bounded on $L^{1}(G)$.

Theorem 1.2 is a straightforward consequence of Thorem 1.1.

Before the proof of Thorem 1.1 we need a lemma about "symbols". We consider it as well-known, but the form given below is adjusted to our needs. 
Lemma 1.3. There is $C$ such that if $E$ is a normed space, $f: \mathbb{R} \longmapsto E$, $|f|$ is integrable, $b \geq 1$,

$$
\begin{gathered}
\sup |\hat{f}| \leq a, \\
\sup \left|\omega \partial_{\omega} \widehat{f}(\omega)\right| \leq a b, \\
\sup \left|\omega^{2} \partial_{\omega}^{2} \widehat{f}(\omega)\right| \leq a b^{2},
\end{gathered}
$$

then

$$
|f(x)| \leq \frac{C a b}{|x|}
$$

REMARK. The lemma remains valid as long as $\hat{f}$ is reasonably defined (like $f \in S\left(\mathbb{R}, E^{*}\right)^{*}$, where $S\left(\mathbb{R}, E^{*}\right)$ consists of $E^{*}$ valued Schwartz class functions).

Proof. Let $\phi \in C^{\infty}(\mathbb{R})$ be such that $\phi(x)=1$ for $|x| \leq 1$ and $\phi(x)=0$ for $|x| \geq 2$. Fix $x_{0} \neq 0$ and let $r=b /\left|x_{0}\right|$. Put $\widehat{f}_{1}(\omega)=\phi(\omega / r) \widehat{f}(\omega)$ and $\widehat{f}_{2}(\omega)=(1-\phi(\omega / r)) \widehat{f}(\omega)$. We have

$$
\left|f_{1}(x)\right| \leq \int\left|\widehat{f}_{1}\right| d \omega \leq \int_{-2 r}^{2 r} a d \omega=4 a r
$$

and

$$
\left|x^{2} f_{2}(x)\right| \leq \int\left|\partial_{\omega}^{2} \widehat{f}_{2}(\omega)\right| d \omega
$$

By the Leibnitz formula

$\partial_{\omega}^{2} \widehat{f}_{2}(\omega)=\left(1-\phi\left(\frac{\omega}{r}\right)\right) \partial_{\omega}^{2} \widehat{f}(\omega)-2 r^{-1} \phi^{\prime}\left(\frac{\omega}{r}\right) \partial_{\omega} \widehat{f}(\omega)+r^{-2} \phi^{\prime \prime}\left(\frac{\omega}{r}\right) \widehat{f}(\omega)$

so

$$
\begin{aligned}
\int\left|\partial_{\omega}^{2} \widehat{f}_{2}(\omega)\right| d \omega \leq & \int_{|\omega|>r} \frac{a b^{2}}{\omega^{2}} d \omega+\int_{2 r>|\omega|>r} 2 C r^{-1} a b \omega^{-1} d \omega \\
& +\int_{2 r>|\omega|>r} C r^{-2} a d \omega \\
\leq & a b^{2} r^{-1}+4 C a b r^{-1}+2 C a r^{-1} \\
\leq & C^{\prime} a b^{2} r^{-1} .
\end{aligned}
$$


Now

$$
\begin{aligned}
\mid f\left(x_{0}\right) & \leq\left|f_{1}\left(x_{0}\right)\right|+\left|f_{2}\left(x_{0}\right)\right| \\
& \leq 4 a r+C^{\prime} a b^{2} r^{-1}\left|x_{0}\right|^{-2} \\
& =\left(4+C^{\prime}\right) a b\left|x_{0}\right|^{-1} \\
& =C^{\prime \prime} a b\left|x_{0}\right|^{-1} .
\end{aligned}
$$

Proof of Theorem 1.1. We decompose the regular representation of $G$ using Fourier transform in $y$ variable. In coordinates

$$
L=-\Delta_{x}-\sum \exp \left(-2 \lambda_{j}(x)\right) \partial_{y_{j}}^{2}
$$

where $\Delta_{x}=\sum \partial_{x_{j}}^{2}$.

If we denote by $H_{z}$ the Fourier transform (in $y$ variable) of $L$ at $z$, then

$$
H_{z}=-\Delta_{x}+\sum z_{j}^{2} \exp \left(-2 \lambda_{j}(x)\right) .
$$

$\operatorname{Re} H_{z} \geq 0$, provided that $\operatorname{Re} z_{j}>\operatorname{Im} z_{j}, j=1, \ldots, m$, so $z \longmapsto$ $\exp \left(-t H_{z}\right)$ is bounded holomorphic in the area given by the inequalities.

Considering $(t+i s) H_{z}$ we see that $\exp \left(-(t+i s) H_{z}\right)$ is bounded and holomorphic as long as $\operatorname{Re}(t+i s) z_{j}^{2} \geq 0, j=1, \ldots, m$. Moreover, we can estimate the integral kernels

$\left\|\exp \left(-(2 t+i s) H_{z}\right) \delta_{0}\right\|_{L^{2}} \leq\left\|\exp \left(-(t+i s) H_{z}\right)\right\|\left\|\exp \left(-t H_{z}\right) \delta_{0}\right\|_{L^{2}}$.

By the Feynmann-Kac formula

$$
\left\|\exp \left(-t H_{z}\right) \delta_{0}\right\|_{L^{2}} \leq\left\|q_{t}\right\|_{L^{2}}=c t^{-n / 4},
$$

where $q_{t}$ is ordinary euclidean heat kernel.

Consequently, by the Cauchy integral formula (for real $z$ )

$$
\left\|\partial_{z}^{\alpha} \exp \left(-(t+i s) H_{z}\right) \delta_{0}\right\|_{L^{2}} \leq C_{\alpha}\left|z_{1}\right|^{-\alpha_{1}} \cdots\left|z_{m}\right|^{-\alpha_{m}}\left(1+\frac{|s|}{t}\right)^{|\alpha|} t^{-n / 4} .
$$

Applying Lemma $1.3 m$ times we get

$$
\|\exp (-(t+i s) L)(\cdot, y)\|_{L^{2}} \leq C^{\prime \prime}\left(\left|y_{1}\right| \cdots\left|y_{m}\right|\right)^{-1}\left(1+\frac{|s|}{t}\right)^{m} t^{-n / 4} .
$$


In [14] (as the first step in proof of Theorem 1.1) we proved that

$$
\int|\exp (-(1+i s) L)(g)| e^{d(g, 0)} d g \leq C \exp \left(C s^{2}\right)
$$

where $d(x, y)$ is the optimal control distance associated to $L$. One easily checks that

$$
\{g: d(g, 0)<r\} \subset\left\{(x, y):|x|<r,|y|<c_{d} \exp \left(c_{d} r\right)\right\}
$$

To estimate $L^{1}$ norm we put $r=C s^{2}, c=c_{d} C, A_{j}=\{(x, y):|x|<$ $\left.C s^{2},\left|y_{j}\right|<\exp \left(-m c s^{2}\right),\left|y_{l}\right|<\exp \left(c s^{2}\right), l \neq j\right\}$. Note $\left|A_{j}\right| \leq C s^{2 n}$. We have

$$
\begin{aligned}
&\|\exp (-(1+i s) L)\|_{L^{1}} \\
& \leq \int_{d(g, 0)>r}|\exp (-(1+i s) L)(g)| d g \\
&+\int_{\substack{|x|<c s^{2} \\
\exp \left(-m c s^{2}\right) \leq\left|y_{j}\right| \leq \exp \left(c s^{2}\right)}}|\exp (-(1+i s) L)((x, y))| d x d y \\
&+\sum_{j} \int_{A_{j}}|\exp (-(1+i s) L)|(g) d g \\
&= I_{\infty}+I_{0}+\sum I_{j}
\end{aligned}
$$

For $I_{\infty}$ we use exponential estimate (1.4)

$$
\begin{aligned}
\int_{d(g, 0)>r} \mid \exp (- & (1+i s) L)(g) \mid d g \\
& \leq e^{-r} \int|\exp (-(1+i s) L)(g)| \exp (d(g, 0)) d g \\
& \leq \exp \left(-C s^{2}\right) C \exp \left(C s^{2}\right)=C .
\end{aligned}
$$

Next

$$
I_{j} \leq\left|A_{j}\right|^{1 / 2}\|\exp (-(1+i s) L)\|_{L^{2}} \leq C|s|^{n} .
$$


Finally

$$
\begin{aligned}
I_{0} & =\int_{\exp \left(-m c s^{2}\right) \leq\left|y_{j}\right| \leq \exp \left(c s^{2}\right)} \int_{|x|<C s^{2}}|\exp (-(1+i s) L)(x, y)| d x d y \\
& \leq \int_{\exp \left(-m c s^{2}\right) \leq\left|y_{j}\right| \leq \exp \left(c s^{2}\right)}\left|\left\{x:|x|<c s^{2}\right\}\right|^{1 / 2} \\
& \cdot\|\exp (-(1+i s) L)(\cdot, y)\|_{L^{2}} d y \\
& \leq \int_{\exp \left(-m c s^{2}\right) \leq\left|y_{j}\right| \leq \exp \left(c s^{2}\right)} c s^{n} C^{\prime \prime}\left(\left|y_{1}\right| \cdots\left|y_{m}\right|\right)^{-1}(1+|s|)^{m} d y \\
& \leq C|s|^{n}(1+|s|)^{m}\left(2 \int_{\exp \left(-m c s^{2}\right)}^{\exp \left(c s^{2}\right)}\left|y_{1}\right|^{-1} d y_{1}\right)^{m} \\
& \leq C|s|^{n}(1+|s|)^{m}\left((m+1) c s^{2}\right)^{m} \\
& \approx C^{\prime}\left(1+|s|^{n+3 m}\right) .
\end{aligned}
$$

\section{Final remarks.}

Our goal was to present the idea, so we used simple arguments even though we got weaker end result. If the estimates are done in a more involved way one may replace $n+3 m$ in Theorem 1.1 by a smaller number (we checked that $(n+3 m) / 2$ is enough), however we expect that in Thorem 1.2 it is enough to have more than $n / 2+m$ derivatives in $L^{2}$, and getting this requires new ideas. Also, constants in Theorem 1.2 grow exponentially with the diameter of support of $F$. We may get polynomial growth, but we would like to have a uniform bound on $\|F(t L)\|_{L^{1}}$

\section{References}

[1] Alexopoulos, G., Spectral multipliers on Lie groups of polynomial growth. Proc. Amer. Math. Soc. 120 (1994), 973-979.

[2] Anker, J. Ph., $L^{p}$ Fourier multipliers on Riemannian symmetric spaces of the non-compact type. Ann. of Math. 132 (1990), 597-628.

[3] Anker, J. Ph., Sharp estimates for some functions of the Laplacian on noncompact symmetric spaces. Duke Math. J. 65 (1992), 257-297. 
[4] Astengo, F., Multipliers for a distinguished Laplacean on solvable extensions of H-type groups. Monatsh. Math. 120 (1995), 179-188.

[5] Christ, M., $L^{p}$ bound for spectral multiplier on nilpotent groups. Trans. Amer. Math. Soc. 328 (1991), 73-81.

[6] Christ, M., Müller, D., On $L^{p}$ spectral multipliers for a solvable Lie group. Geom. Funct. Anal. 6 (1996), 860-876.

[7] Christ, M., Sogge, Ch., The weak type $L^{1}$ convergence of eigenfunction expansions for pseudodifferential operators. Invent. Math. 94 (1988), 421-453.

[8] Clerc, J. L., Stein, E. M., $L^{p}$-multipliers for non-compact symmetric spaces. Proc. Nat. Acad. Sci. USA (1974), 3911-3912.

[9] Cowling, M., Giulini, S., Hulanicki, A., Mauceri, G., Spectral multipliers for a distinguished Laplacian on certain groups of exponential growth. Studia Math. 111 (1994), 103-121.

[10] Hebisch, W., The subalgebra of $L^{1}(A N)$ generated by the laplacean. Proc. Amer. Math. Soc. 117 (1993), 547-549.

[11] Hebisch, W., Multiplier theorem on generalized Heisenberg groups. Coll. Math. 65 (1993), 231-239.

[12] Hebisch, W., Zienkiewicz, J., Multiplier theorem on generalized Heisenberg groups II. Coll. Math. 69 (1995), 29-36.

[13] Hebisch, W., Boundedness of $L^{1}$ spectral multipliers for an exponential solvable Lie group. Coll. Math. 73 (1997), 155-164.

[14] Hebisch, W., Spectral multipliers on exponential growth solvable Lie groups. Math. Z. 229 (1998), 435-441.

[15] Hörmander, L., Estimates for translation invariant operators in $L^{p}$ spaces. Acta Math. 104 (1960), 93-140.

[16] Hulanicki, A., Subalgebra of $L_{1}(G)$ associated with Laplacian on a Lie group. Colloq. Math. 31 (1974), 259-287.

[17] Ludwig, J., Müller, D., Sub-Laplacians of holomorphic $L^{p}$-type on rank one $A N$-groups and related solvable groups. J. Funct. Anal. 170 (2000), 366-427.

[18] Mauceri, G., Meda, S., Vector-valued multipliers on stratified groups. Revista Mat. Iberoamericana 6 (1990), 141-154.

[19] Müller, D., Stein, E. M., On spectral multipliers for Heisenberg and related groups. J. Math. Pures et Appl. 73 (1994), 413-440.

[20] Mustapha, S., Multiplicateurs spectraux sur certains groupes non-unimodulaires, Harmonic Analysis and Number Theory. CMS Conf. Proceedings, Vol. 21, 1997.

[21] Mustapha, S., Multiplicateurs de Mikhlin pour une classe particulière de groupes non-unimodulaires. Ann. Inst. Fourier 48 (1998), 957-966. 
[22] Sikora, A., Multiplicateurs associés aux souslaplaciens sur les groupes homogènes. C. R. Acad. Sci. Paris 315 (1992), 417-419.

[23] Taylor, M., $L^{p}$-estimate on functions of the Laplace operator. Duke Math. J. 58 (1989), 773-793.

Recibido: 17 de septiembre de 1.999

Revisado: 7 de diciembre de 1.999

\author{
Waldemar Hebisch ${ }^{1}$ \\ Institute of Mathematics \\ Wrocław University \\ pl. Grunwaldzki 2/4 \\ 50-384 Wrocław, POLAND \\ hebisch@math.uni.wroc.pl
}

1 Partially supported by KBN grant 2 P03A 05814 and European Commision via TMR network "Harmonic analysis" 